\title{
PML overexpression inhibits proliferation and promotes the osteogenic differentiation of human mesenchymal stem cells
}

\author{
JIE SUN* ${ }^{*}$ SHAN FU*, WEIJUN ZHONG and HE HUANG \\ The First Affiliated Hospital, Medical School of Zhejiang University, Hangzhou 310003, P.R. China
}

Received August 23, 2013; Accepted September 23, 2013

DOI: $10.3892 /$ or.2013.2786

\begin{abstract}
The promyelocytic leukemia (PML) gene, as an important tumor-suppressor, has been proven to regulate stem cell function in multiple tissues; however its role in human mesenchymal stem cells (hMSCs) remains unclear. In the present study, the effect of PML on regulating the proliferation and osteogenic differentiation of hMSCs was explored. New downstream genes that may be responsible for the regulation of PML were found, and possible mechanisms were analyzed. The lentiviral vector which encodes full-length human PML cDNA or shRNA against PML was transfected into hMSCs. RT-PCR and western blotting were used to detect mRNA and protein expression. Flow cytometry was used to analyze apoptosis and the cell cycle distribution. Osteogenic differentiation of hMSCs was induced by osteo-inductive medium for 7 to 14 days. cDNA microarray was used to scan the gene expression profile and to identify significant changes in gene expression. In the present study, we found that PML was stably expressed in hMSCs, and the expression was increased time-dependently along with cell osteogenic differentiation. Overexpression of PML inhibited hMSC proliferation by inducing apoptosis and arresting the cell cycle. However, PML enhanced the osteoblast differentiation potential of hMSCs. PML-overexpressing hMSCs had a significant increase in mineralized matrix production and ALP activity on day 7 under osteogenic or non-osteogenic differentiation conditions. Upregulation of integrin-binding sialoprotein (IBSP, bone sialoprotein) induced by PML overexpression was found. Our data indicate that PML regulates hMSCs as an inhibitor of cell proliferation but a promoter of osteogenic differentiation.
\end{abstract}

Correspondence to: Professor He Huang, The First Affiliated Hospital, Medical School of Zhejiang University, Hangzhou 310003, P.R. China

E-mail: hehuang.zju@gmail.com

${ }^{*}$ Contributed equally

Key words: promyelocytic leukemia gene, cell proliferation, osteogenic differentiation, human mesenchymal stem cells

\section{Introduction}

Human mesenchymal stem cells (hMSCs) are an important type of adult stem cell and have multiple differentiation activities and high self-renewal potential. The ease of isolation and availability of hMSCs make them attractive tools for potential use in cell transplantation therapy and tissue repair $(1,2)$. However, the mechanism of the self-renewal mechanisms and multiple differentiation capabilities of hMSCs have not been fully elucidated, thus limiting the use of these powerful tools.

Recently, many research groups have investigated the regulation of hMSC proliferation and differentiation, and several important regulatory pathways have been revealed. For instance, the role of the Wnt (wingless) signaling pathway on the fate of hMSCs has been established. However, the effects of Wnt signaling can induce different or even opposite biological functions (1,3). In the differentiation process of hMSCs, the role of Wnt signaling is dependent on the level of the signals, and high levels of Wnts promote osteogenesis of hMSCs, whereas low levels inhibit osteogenesis (4). Thus, the complex effect of Wnt signaling is context-dependent and is closely related to its target genes (3). Furthermore, Shtutman et al (5) reported that the promyelocytic leukemia (PML) gene can function as a target of the Wnt signaling pathway, and its gene product is involved in the regulation of various cell functions. Thus, the role of the PML protein in hMSCs aroused our interest.

PML, as an important tumor-suppressor, was initially discovered in patients with acute promyelocytic leukemia (APL) (6). In normal cells, PML is an important regulator of numerous fundamental processes, such as apoptosis, cellular proliferation, senescence and cell cycle regulation. Recently, PML was found to regulate neural progenitor cells (NPCs), leukemia-initiating cells (LICs) and hematopoietic stem cells (HSCs) (7-9). Loss of PML increases the number of NPCs and can impair their differentiation. PML is highly expressed in HSCs and is responsible for normal cellular function and maintenance, while its deficiency results in progressive impairment of cell long-term repopulating capacity (9). However, the role of PML in regulating hMSCs remains unclear.

In the present study, we investigated the regulation of PML in hMSC proliferation and the progression of osteoblast differentiation. The possible underlying mechanisms involved in the regulation of proliferation and differentiation of hMSCs were also explored. 


\section{Materials and methods}

Cell isolation and culture. The present study was approved by the Ethics Committee of our hospital, and informed consent was obtained from each patient prior to obtaining bone marrow samples. hMSCs were obtained from the bone marrow of patients undergoing total hip replacement surgeries. The mononuclear cells were then isolated by density gradient centrifugation. Cells were maintained in low-glucose Dulbecco's modified Eagle's medium (L-DMEM) supplemented with $10 \%$ fetal bovine serum (FBS) (both from Gibco-BRL, Carlsbad, CA, USA), which was replaced every 3 days. When cells reached $70-80 \%$ confluence, they were passaged by trypsinization. All cells were grown in a monolayer culture in a humidified incubator containing $5 \% \mathrm{CO}_{2}$ at $37^{\circ} \mathrm{C}$. Flow cytometry was used to identify the phenotype of the hMSCs, and those in the third passage from the same batch were used in the following experiments. Every experiment was repeated using 3 different hMSCs strains isolated from 3 different randomly chosen samples.

Cell proliferation analysis. Cell viability was determined using the Cell Counting Kit-8 assay (CCK-8; Dojin, Tokyo, Japan). Briefly, cultured cells were plated at a density of 3,000 cells/well in 96 -well plates and incubated at $37^{\circ} \mathrm{C}$ in a humidified incubator containing $5 \% \mathrm{CO}_{2}$ for $0,1,3,5$, or 7 days. At the indicated time points, cells were incubated with $20 \mu \mathrm{l}$ of CCK-8 solution for $2 \mathrm{~h}$ at $37^{\circ} \mathrm{C}$, and the absorbance at $490 \mathrm{~nm}$ was measured using a microplate reader (Bio-Rad Laboratories, Tokyo, Japan). Three contiguous wells were used for each testing group.

Flow cytometric analysis of the cell cycle and apoptosis. After the designated treatments, the adherent and supernatant cells were harvested. For cell cycle analysis, the cells were harvested, washed with phosphate-buffered saline (PBS) containing $0.1 \%$ bovine serum albumin (BSA), fixed with $70 \%$ ethanol for $2 \mathrm{~h}$, and washed twice with PBS. Then cells were stained with $0.5 \mathrm{ml} \mathrm{PI} / \mathrm{RNase}$ staining buffer for $15 \mathrm{~min}$ at room temperature (BD Biosciences, San Diego, CA, USA). Apoptosis analysis was performed using the BD Pharmingen ${ }^{\mathrm{TM}}$ PE Annexin V Apoptosis Detection kit (BD Biosciences). Briefly, the cells were washed and re-suspended in $500 \mu \mathrm{l}$ of binding buffer, then incubated with $5 \mu \mathrm{l}$ of Annexin V-PE and $5 \mu 1$ of 7-amino-actinomycin D (7-AAD) solution for $15 \mathrm{~min}$ in the dark at room temperature. The DNA content and cell apoptosis were then analyzed by flow cytometry (FC500; Beckman Coulter, Inc., Brea, CA, USA). Three contiguous wells were used for each testing group.

Induction of osteogenic differentiation. For osteogenic differentiation, cells were plated in 6-well plates at a density of 50,000 cells/well. When the cells reached $70 \%$ confluence, they were treated with or without osteogenic supplements (10 $\mathrm{nM}$ dexamethasone, $10 \mathrm{mM} \beta$-glycerophosphate and $50 \mu \mathrm{g} / \mathrm{ml} \mathrm{L}$-ascorbic acid-2-phosphate; all from Sigma-Aldrich, Poole, UK) for 7 or 14 days. The medium was replaced every 3 days. Cells were verified by von Kossa staining and alkaline phosphatase (ALP) activity analysis. ALP activities were normalized to total protein content using the BCA protein assay kit (Beyotime, Jiangsu, China) and were expressed as units/mg protein. Three contiguous wells were used for each testing group.

Immunofluorescence staining. hMSCs at passage 3 were seeded on glass coverslips in 6-well plates at a density of $3 \times 10^{4} /$ well. For immunofluorescence staining, the coverslips were removed and washed twice with PBS, fixed with $4 \%$ paraformaldehyde for $15 \mathrm{~min}$, and then permeabilized with $0.2 \%$ Triton X-100 for $10 \mathrm{~min}$ at room temperature. Next, the cells were washed 3 times with PBS, blocked with $10 \%$ BSA at room temperature for $60 \mathrm{~min}$, and then incubated at $4^{\circ} \mathrm{C}$ overnight with the rabbit anti-PML polyclonal antibody (1:500 dilution; Abcam, Hong Kong). The cells were then washed 3 times with PBS and incubated for $30 \mathrm{~min}$ at room temperature with fluorescein isothiocyanate-conjugated anti-rabbit antibodies (1:1,000 dilution; Boster Biological Technology, Wuhan, China). The cells were washed 3 times, stained with 4'6'-diamidino-2-phenylindole (DAPI) $(10 \mu \mathrm{g} / \mathrm{ml})$ for $3 \mathrm{~min}$ and then photographed via fluorescence microscopy.

Lentiviral vectors and hMSC transduction. Recombinant lentiviral vector plenti6.3-PML-EGFP which encodes the full-length human PML-cDNA (NM_002675) and the enhanced green fluorescent protein (EGFP) was synthesized by Invitrogen Life Technologies (Shanghai, China). The empty vector, plenti6.3-EGFP, was used as the control. The lentiviruses were produced as described previously. Briefly, human embryonic kidney 293T cells were used as packaging cells. One day before transfection, the $293 \mathrm{~T}$ cells were seeded at a density of $5 \times 10^{6}$ cells $/ 10-\mathrm{cm}$ dish and cultured to $90-95 \%$ confluence. Plenti6.3-PML-EGFP or control vectors $(3 \mu \mathrm{g})$, together with packaging plasmids ( $9 \mu \mathrm{g}$; pLP1, pLP2 and pLP/VSVG), were cotransfected into $293 \mathrm{~T}$ cells using Lipofectamine ${ }^{\mathrm{TM}} 2000$ (both from Invitrogen Life Technologies) according to the manufacturer's instructions. After $48 \mathrm{~h}$, the cell culture supernatant was collected and passed through a $0.45-\mu \mathrm{m}$ filter. When the hMSCs reached 50-60\% confluence, they were transfected with either the PML or control lentivirus at a variety of multiplicity of infections (MOIs) ranging from 0-100. After incubation for $72 \mathrm{~h}$, the transfection efficiency was estimated by evaluation of EGFP expression via fluorescence microscopy, and PML gene expression was verified by quantitative real-time PCR (qRT-PCR) and western blot analysis.

Short hairpin RNA (shRNA) preparation and gene transfection. Four different shRNAs against PML in a lentiviral vector with green fluorescent protein and the control vector were designed and synthesized by GenePharma Inc. (Shanghai, China). Prior to lentivirus-mediated knockdown of PML in hMSCs, the inhibitory activity of the shRNAs was tested in 293T cells, and the most effective sequence was used for transfection into the hMSCs. As described earlier, high-titer lentivirus was produced in 293T cells by transfection of the lentiviral expression vector and packaging vectors. The lentivirus was harvested after $48 \mathrm{~h}$, filtrated and was used to infect hMSCs which had reached 50-60\% confluence. After transfection for $72 \mathrm{~h}$, the efficiency was estimated by evaluation of EGFP expression via fluorescence microscopy, and PML gene expression was verified by qRT-PCR and western blot analysis. 
The DNA sequences of the PML shRNA was 5'-TGGAGGAG GGTTCCAGTTTCTTTCAAGAGAAGAAACTGGAACTC CTCCTCСTTTTTTC-3' and 5'-TCGAGAAAAAAGGAGG AGGAGTTCCAGTTTCTTCTCTTGAAAGAAACTGGAA CTCCTCCTCCA-3'. The control shRNA was 5'-TGTTCTCC GAACGTGTCACGTTTCAAGAGAACATGACACGTTCG GAGAACTTTTTTC-3' and 5'-TCGAGAAAAAAGTTCTC CGAACGTGTCACGTTCTCTTGAAACGTGACACGTTC GGAGAACA-3'.

Reverse transcription and real-time PCR analysis. Total cellular RNA was extracted using TRIzol reagent (Invitrogen Life Technologies), and $1 \mu \mathrm{g}$ RNA was reverse-transcribed to cDNA using Moloney murine leukemia virus (MMLV) reverse transcriptase (Fermentas-Thermo Fisher Scientific, Inc., Waltham, MA, USA). Qualitative analysis of RNA expression was carried out by reverse transcription-polymerase chain reaction (RT-PCR) using $\beta$-actin mRNA as the internal control. cDNA was amplified in a final reaction volume of $20 \mu 1$ using a Takara $\mathrm{Taq}^{\mathrm{TM}}$ kit. The amplification consisted of 35 cycles for $30 \mathrm{sec}$ at $95^{\circ} \mathrm{C}, 45 \mathrm{sec}$ at $59^{\circ} \mathrm{C}$ and $1 \mathrm{~min}$ at $72^{\circ} \mathrm{C}$. Then, the PCR products were electrophoresed on a $1.5 \%$ agarose gel containing ethidium bromide and visualized by UV absorption.

Quantitative analysis of RNA expression was carried out by qRT-PCR. The cDNA amplification was performed in a total volume of $20 \mu \mathrm{l}$ in 40 cycles of $95^{\circ} \mathrm{C}$ for $5 \mathrm{sec}$, and $59^{\circ} \mathrm{C}$ for $20 \mathrm{sec}$ using the LightCycler RT-PCR system (Roche, Mannheim, Germany) according to the manufacturer's instructions. Data were analyzed using the relative standard curve method, and each sample was normalized to $\beta$-actin to correct differences in RNA quality and quantity. In each experiment, every sample was assayed in triplicate, and each experiment was performed 3 times. The forward and reverse primers for amplification of PML, ALP, IBSP, and $\beta$-actin are as follows: PML forward, 5'-TGTACCG GCAGATTGTGGAT-3' and reverse, 5'-AGATGTTGTTGGT CTTGCGG-3'; ALP forward, 5'-GACCATTCCCACGTCTT CACATT-3' and reverse, 5'-CAGACTGCGCCTGGTAGTT GT-3'; IBSP forward, 5'-CCAGAGGAAGCAATCACCA AA-3' and reverse, 5'-TTGAGAAAGCACAGGCCATTC-3'; $\beta$-actin forward, 5'-AGCGAGCATCCCCCAAAGTT-3' and reverse, 5'-GGGCACGAAGGCTCATCATT-3'.

Western blot analysis. hMSCs proteins were lysed with radioimmunoprecipitation assay (RIPA) buffer and proteinase inhibitors (Beyotime). Protein concentrations were determined using the bicinchoninic acid protein assay with BSA as a standard (Beyotime). Equal amounts of cell proteins $(30 \mu \mathrm{g})$ were subjected to $10 \%$ SDS-polyacrylamide gel electrophoresis and electrotransferred onto polyvinylidene difluoride (PVDF) membranes, which were blocked with $5 \%$ phosphatase-free powdered milk in Tris-buffered saline with $0.1 \%$ Tween (TBST) for $2 \mathrm{~h}$. Then, the membranes were incubated overnight at $4^{\circ} \mathrm{C}$ with the primary antibodies consisting of PML (Abcam), caspase-3, poly(ADP-ribose) polymerase (PARP) and $\beta$-actin antibody (Cell Signaling Technology, Inc.). The membranes were then rinsed with TBST and incubated with horseradish peroxidase-conjugated goat anti-mouse or goat anti-rabbit immunoglobulin $\mathrm{G}$ antibody (Abcam) for $1.5 \mathrm{~h}$.
The membranes were exposed to X-ray film, and the density of each band was quantified using ImageJ software (National Institutes of Health). Expression of the protein of interest was normalized to $\beta$-actin expression in the same lane. All experiments were repeated at least 3 times and each yielded similar results.

Microarray and data analyses. The microarray analyses were performed by Kangchen Biotech Co., Ltd. (Shanghai, China) using standard protocols. Briefly, when PML was overexpressed for 3 days, total RNA from normal, mock-, or PML-transfected hMSCs was isolated using TRIzol reagent. Double-stranded cDNA was synthesized from total RNA using the SupersScript ${ }^{\circledR}$ Double-Stranded cDNA Synthesis kit (Invitrogen Life Technologies) and labeled in accordance with the NimbleGen Gene Expression Analysis protocol (Roche NimbleGen Systems, Inc., Madison, WI, USA).

An Axon GenePix 4000B scanner (Molecular Devices, Inc., Downingtown, PA, USA) and NimbleScan software (version 2.5) was used to analyze the expression data, which were normalized through quantile normalization using the Robust Multichip average algorithm included in the NimbleScan software. All gene level files were imported into GeneSpring GX software (version 11.5.1; Agilent Technologies, Inc., Santa Clara, CA, USA) for further analysis. Differentially expressed genes with statistical significance were identified through volcano plot filtering and hierarchical clustering using the Agilent GeneSpring GX software.

Statistical analysis. All statistical analyses were performed using SPSS software (SPSS, Inc., Chicago, IL, USA), and all data are expressed as means \pm standard deviation. Differences between groups were evaluated using one-way analyses of variance (ANOVA), and a P-value $<0.05$ was considered to indicate a statistically significant result.

\section{Results}

Phenotypes of the purified bone marrow cells. The hMSCs exhibited typical spindle shapes, and significant colonies were observed on day 7 of the primary culture (Fig. 1A). After reaching confluence, the cells were arranged in various orientations and exhibited vortex-like growth (Fig. 1B). The hMSCs phenotypes were identified by flow cytometry which demonstrated that they were uniformly positive for cluster of differentiation CD29, CD44 and CD166, but negative for CD34, CD45 and human leukocyte antigen DR (data not shown), consistent with the cytobiological characteristics reported in our previous studies and others (10-12). Isolated hMSCs were successfully transformed into osteoblasts, as indicated by positive von Kossa staining after 14 days of induction (Fig. 1C).

PML is stably expressed in hMSCs and expression is increased time-dependently along with cell osteogenic differentiation. PML was positively expressed in the hMSCs, and both mRNA and protein concentrations were detectable over 9 days along with cell proliferation (Fig. 2A). The induction of hMSCs to differentiate into osteoblasts increased the expression of PML, which was consistent with the upregulation of ALP mRNA in 

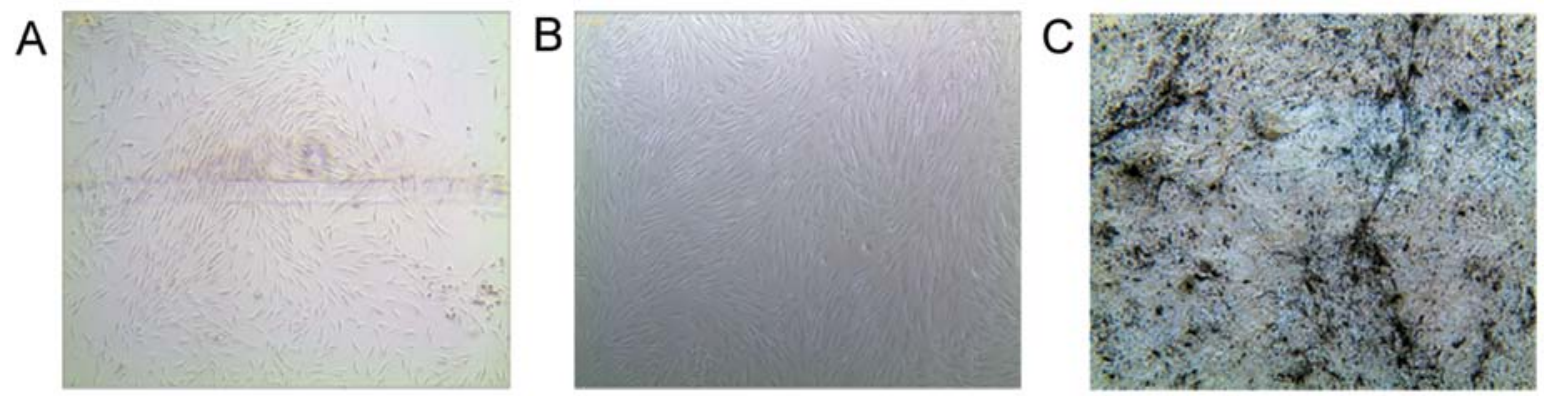

Figure 1. Human mesenchymal stem cells purified from bone marrow. (A) Significant colonies were observed on day 7 of the primary culture and (B) passage 3 (P3) hMSCs had various orientations and exhibited vortex-like growth. (C) hMSCs were successfully induced into osteoblasts and were positive for von Kossa staining after 14 days of induction. Scale bar, $100 \mu \mathrm{M}$. hMSCs, human mesenchymal stem cells.
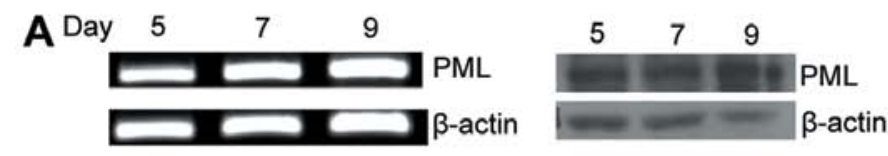

C
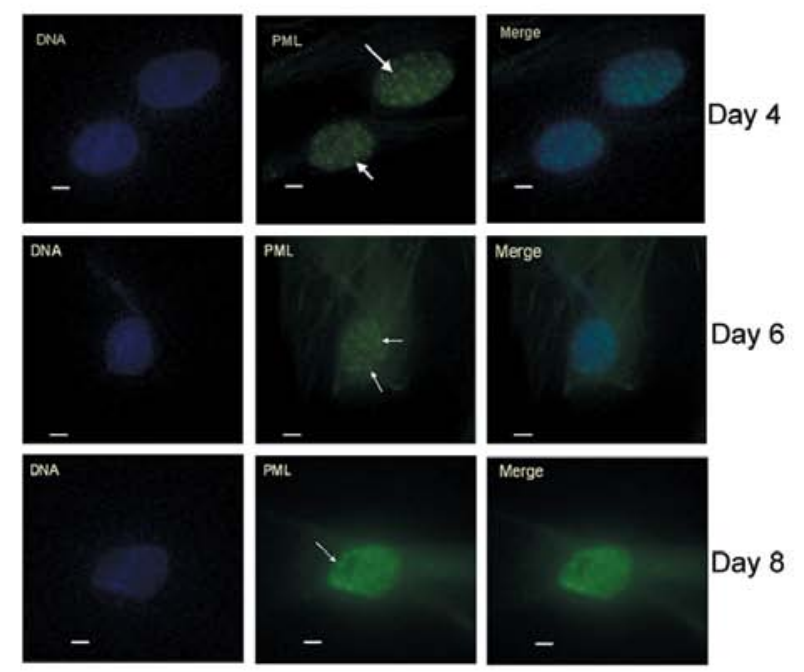
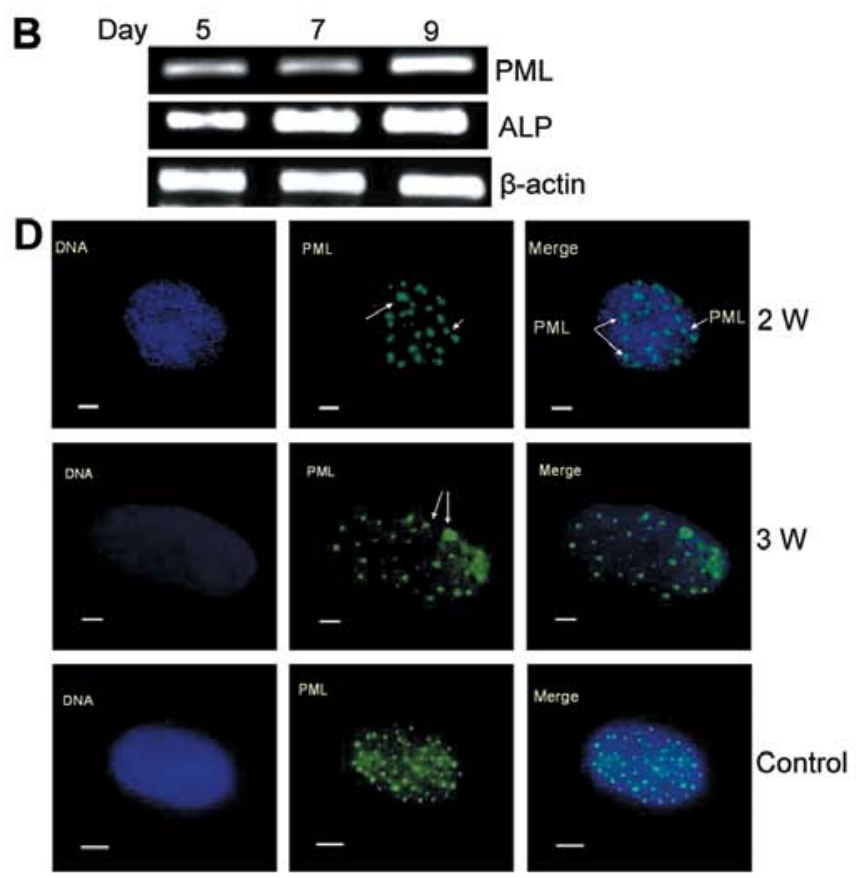

Figure 2. PML expression in hMSCs and the correlation of PML with cell proliferation and osteogenic differentiation. (A) PML was positively expressed in the hMSCs, and both mRNA and protein concentrations were detectable over 9 days along with cell proliferation. (B) During the osteogenic differentiation process, expression of PML was increased, which was consistent with the upregulation of ALP mRNA in a time-dependent manner. (C) During hMSCs proliferation, the location of PML-NBs exhibited no significant change as detected by immunofluorescence staining on days 4,6 and 8 . (D) PML-NBs became larger and unequal in size as detected by immunofluorescence staining after 2 and 3 weeks of osteogenic differentiation. Normal cells were used as control. PML, promyelocytic leukemia; hMSCs, human mesenchymal stem cells; PML-NBs, PML-nuclear bodies.

a time-dependent manner (Fig. 2B). The PML protein epitomizes the PML-nuclear body (PML-NB), which is involved in the regulation of cell function, and the relative size of PML-NBs can change depending on cell status. PML-NBs in hMSCs were not significantly altered along with cell proliferation as detected by immunofluorescence staining on day 4, 6 and 8 (Fig. 2C). However, when cells were induced to differentiate into osteoblasts for 2 and 3 weeks, the PML-NBs became larger and unequal in size when compared to those of normal cells (Fig. 2D). Our preliminary results showed that PML was stably expressed in hMSCs and was associated with osteogenic differentiation of hMSCs.

PML overexpression in hMSCs by stable transduction. In order to determine the impact of PML on hMSCs proliferation and differentiation, a lentiviral vector encoding full-length human PML cDNA was transfected into hMSCs. A vacant vector was transfected as a negative control, and non-transfected hMSCs served as normal controls. At an MOI of 40, $>80 \%$ of the hMSCs were EGFP-positive on day 3, as assessed by fluorescence microscopy (Fig. 3A), and flow cytometry. At the same time, PML expression in transfected hMSCs was detected by qRT-PCR and western blotting. As shown in Fig. 3B, PML RNA expression was significantly increased in PML-transfected cells when compared with that in the untreated and mock-transfected cells, and the PML protein was readily detected in the PML-transfected cells, but was very low in the untreated and mock-transfected cells, respectively (Fig. 3C).

PML overexpression inhibits hMSCs proliferation. PML overexpression in hMSCs induced a significant decrease in cell 
A
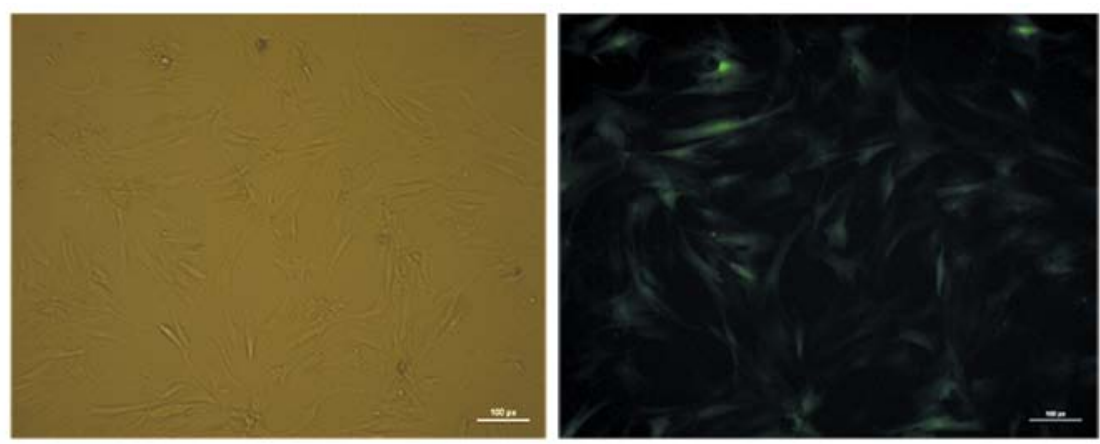

B

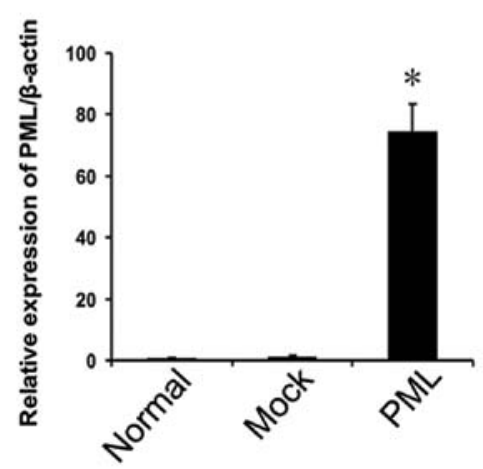

C

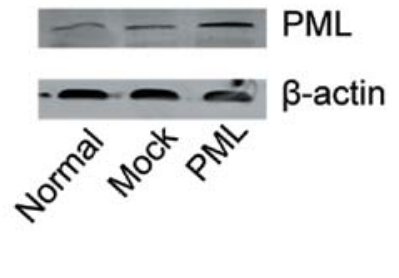

Figure. 3. PML overexpression in hMSCs by stable transduction. (A) hMSCs were positive for EGFP on day 3 after lentiviral infection, as detected by fluorescence microscopy. Scale bar, $100 \mu \mathrm{M}$. On day 3, (B) PML mRNA and (C) protein expression was significantly increase in the PML-transfected cells when compared with that in the untreated and mock-transfected cells. ${ }^{*} \mathrm{P}<0.05$ vs. the two control groups. PML, promyelocytic leukemia; hMSCs, human mesenchymal stem cells; EGFP, enhanced green fluorescent protein.

proliferation as indicated by the change in absorbance from day 0 to day 7 (Fig. 4A). Cell proliferation was inhibited by $20.57 \pm 4.18 \%$ in $\mathrm{hMSCs}$ overexpressing PML as compared to that of the normal controls $(\mathrm{P}<0.05)$ and by $18.46 \pm 3.53 \%$ when compared to the mock-transfected cells on day $3(\mathrm{P}<0.05)$. PML overexpression also significantly increased cell apoptosis. After 3 days of PML overexpression, the percentage of early apoptotic cells in the PML-transfected cells was $10.3 \pm 4.23 \%$, which was significantly higher than that in the mock-transfected cells $(2.53 \pm 0.77 \%)(\mathrm{P}<0.05)$ and normal control cells $(2.18 \pm 1.88 \%)(\mathrm{P}<0.05)$ (Fig. 4B). Western blot analysis showed that this process occurred concomitantly with a decrease in production of the apoptosis marker proteins procaspase- 3 and PARP, and an increase in production of cleaved caspase-3 (Fig. 4C). There were no obvious changes to the cell cycle (data not shown) when PML expression was enhanced. However, when the expression of PML was knocked down for 3 days (Fig. 4D), the proportion of hMSCs in the S/G2+M phases was clearly increased (Fig. 4E).

PML overexpression promotes osteogenic differentiation of hMSCs. To evaluate the effects of PML overexpression on the osteogenic differentiation of hMSCs, PML-transfected cells were induced to osteogenic differentiation, while normal and mock-transfected cells served as controls. von Kossa staining and ALP activity were used to evaluate the degree of osteogenic differentiation. As shown in Fig. 5A, mineralized matrix production was strongly enhanced on day 7 in the PML-overexpressing hMSCs under osteogenic differentiation conditions and the ALP activity was obviously increased in comparison to the mock-transfected and normal MSCs
$(0.39 \pm 0.019$ vs. $0.24 \pm 0.016$ and $0.2 \pm 0.008 \mathrm{U} / \mathrm{mg}$, respectively) ( $\mathrm{P}<0.05$ for both) (Fig. 5B). Next, calcium deposition and ALP activity were detected in PML-transfected cells under non-osteogenic differentiation conditions (DMEM-LG containing 10\% FBS). We found that PML-overexpressing hMSCs exhibited an obvious increase in mineralized matrix production on day 7 when compared with that in the normal and mock-transfected cells (Fig. 5A). Under normal culture conditions for 7 days, ALP activity was also obviously increased in comparison to the mock-transfected and normal MSCs $(0.049 \pm 0.006$ vs. $0.024 \pm 0.005$ and $0.028 \pm 0.005 \mathrm{U} / \mathrm{mg}$, respectively) ( $\mathrm{P}<0.05$ for both) (Fig. 5B). Next, alterations in ALP mRNA expression were investigated by RT-PCR. mRNA expression of ALP as an early marker of osteoblastic differentiation, was dramatically enhanced on day 7 in PML-overexpressing hMSCs. Knockdown of PML expression decreased ALP mRNA expression when the cells were cultured under normal conditions for 7 days (Fig. 5C). Together, these findings suggest that PML promotes the osteogenic differentiation potential of hMSCs even under non-osteogenic differentiation conditions.

Typical gene expression from the microarray and confirmation . To explore the potential mechanism of PML in the regulation of hMSC proliferation and differentiation, we investigated genome-wide expression profiles of the normal, mock- and PML-transfected hMSCs. The microarray employed for the present analysis consisted of 45,033 genes that were collected from genetic databases, including that from the National Center for Biotechnology Information. After performing t-test analyses, a total of 357 genes were found to have 
A

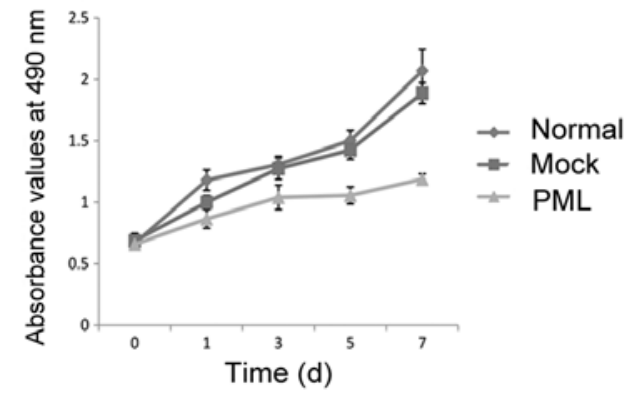

B

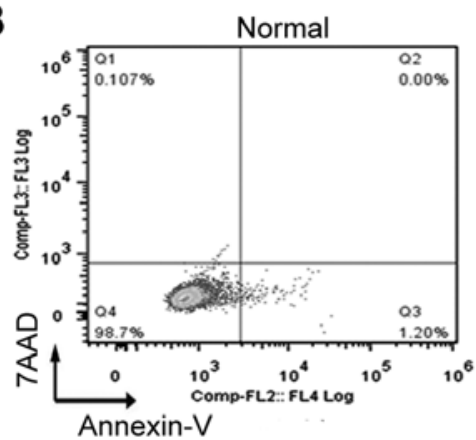

C

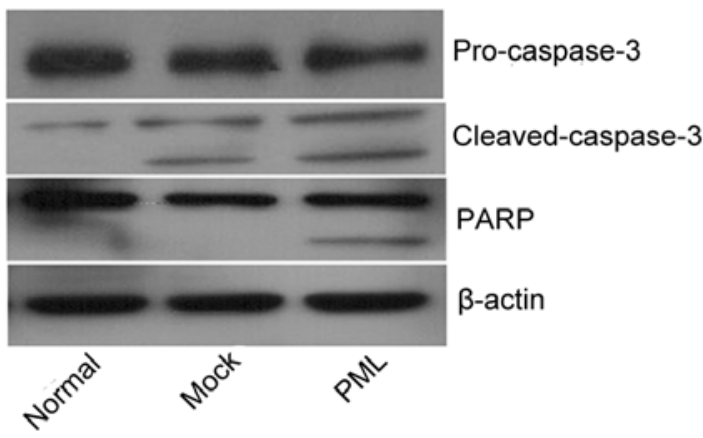

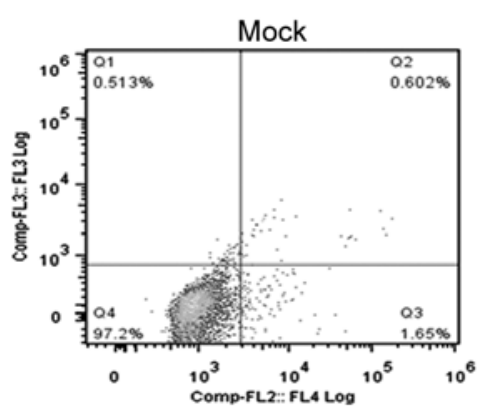

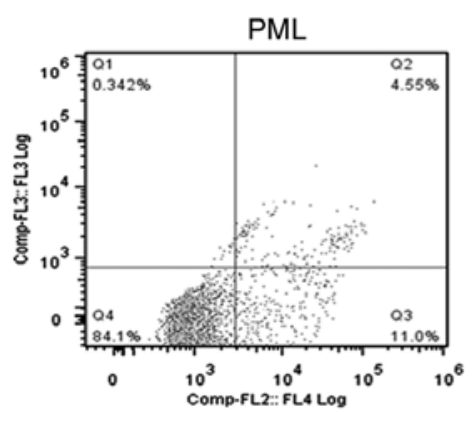

\section{E}

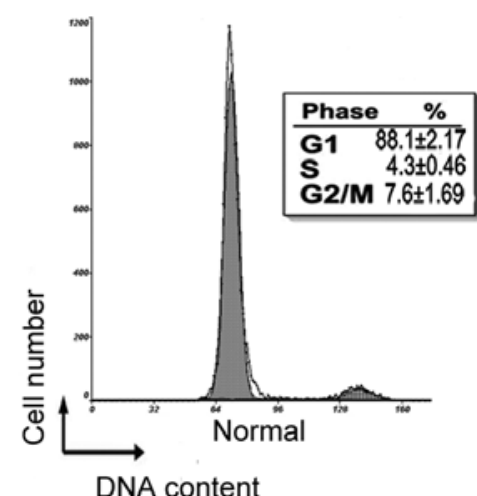

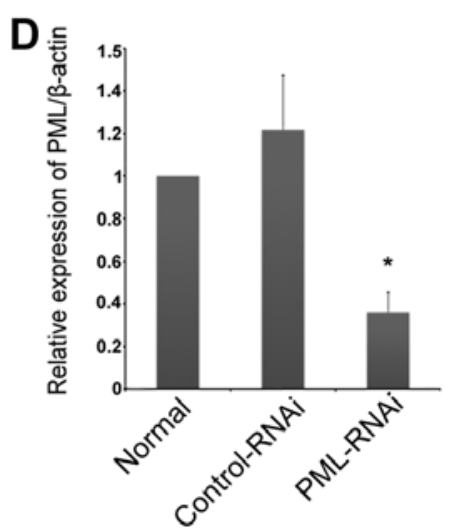

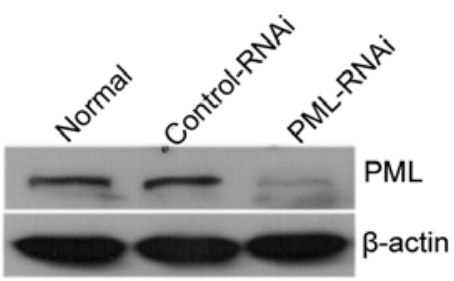

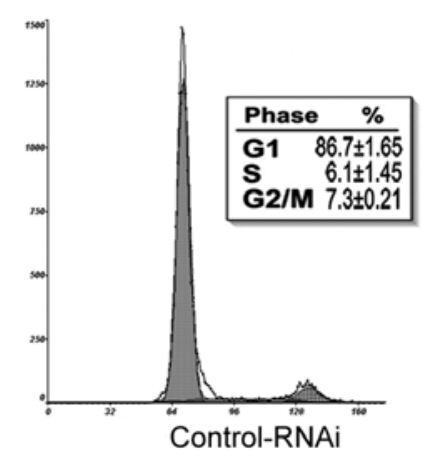

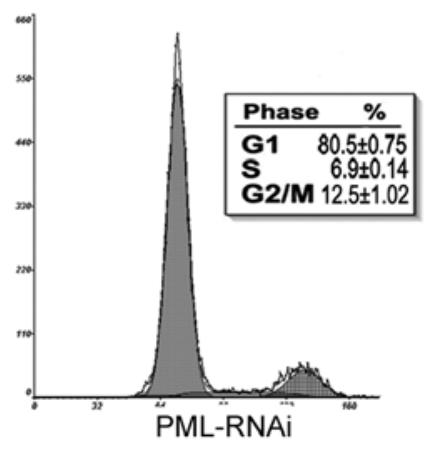

Figure 4. PML overexpression inhibits hMSC proliferation. (A) Growth curves of normal, mock- and PML-transfected hMSCs as measured via the CCK-8 assay on days $0,1,3,5$ and 7. (B) Cell apoptosis was detected by flow cytometry in normal, mock- and PML-transfected cells on day 3 after lentiviral infection (C) Apoptosis-associated proteins were detected by western blotting. Enhanced PML expression induced caspase-associated apoptosis. (D) PML mRNA and protein expression was detected in hMSCs transfected with PML-shRNA for 3 days. (E) Cell cycle distribution was evaluated using flow cytometry. Loss of PML expression promoted cell cycle progression. ${ }^{*} \mathrm{P}<0.05$ vs. the two control groups. PML, promyelocytic leukemia; hMSCs, human mesenchymal stem cells; CCK-8, Cell Counting Kit-8.

significantly altered expression levels of $>1.5$-fold $(\mathrm{P}<0.05)$, of which 222 were upregulated and 135 were downregulated in the PML-transfected hMSCs when compared to the normal and mock-transfected cells. In the PML-transfected cells, the 3 most upregulated genes were interleukin (IL)-1 $\beta$, IL-8 and IBSP, while the most downregulated gene was fibroblast growth factor receptor 2 (FGFR2) (Table I). IBSP, as an important marker of osteogenic differentiation, plays a key role in the regulation of osteogenic differentiation. Next, we validated the IBSP expression levels by qRT-PCR. Previous 
A

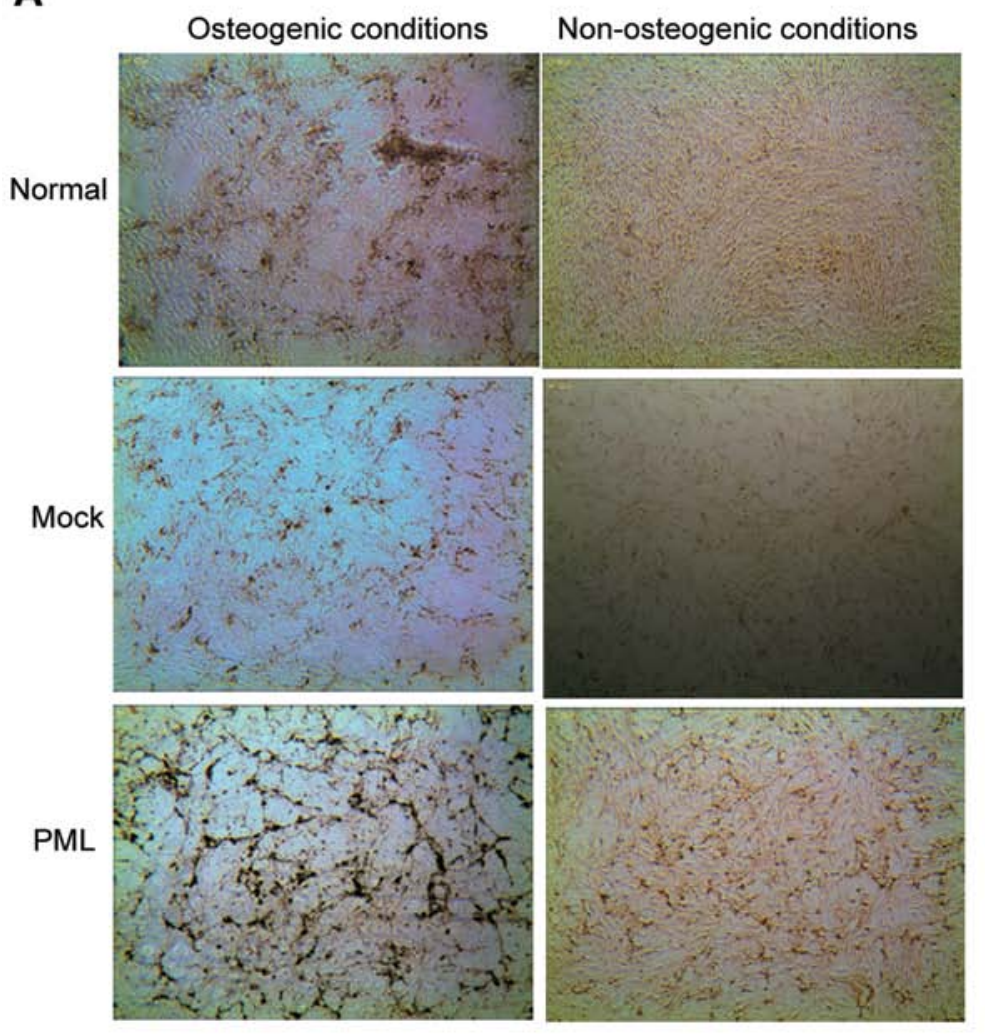

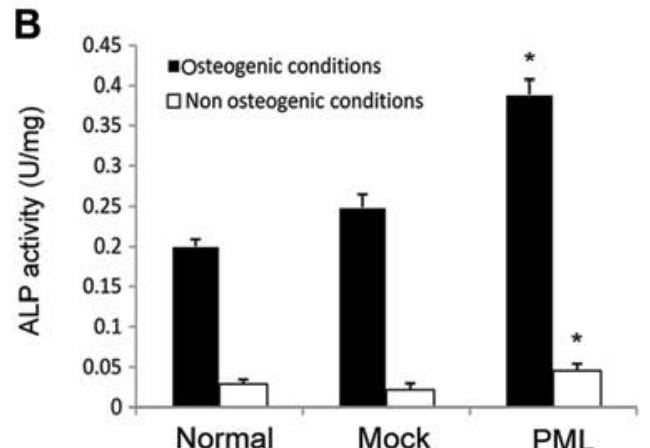

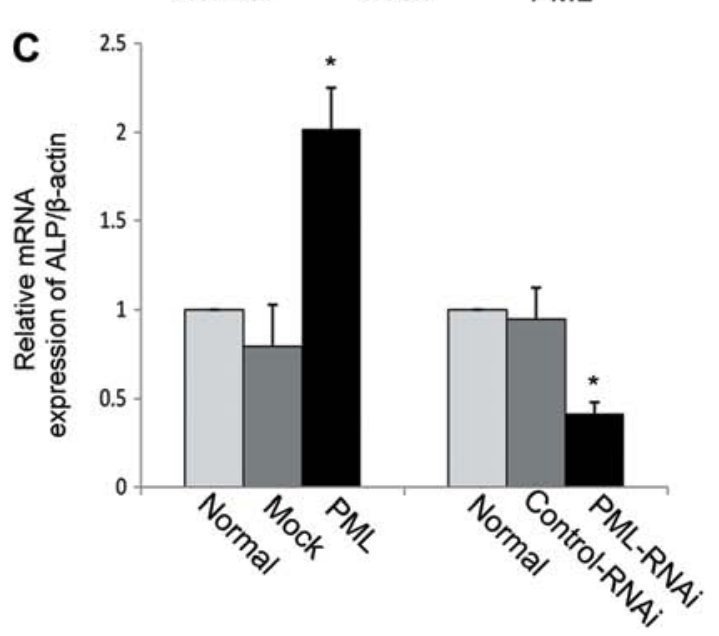

Figure 5. PML promotes the osteogenic differentiation potential of hMSCs. (A) Calcium deposition of hMSCs as detected by von Kossa staining. Mineralized matrix production was strongly enhanced on day 7 in PML-overexpressing hMSCs under osteogenic or non-osteogenic differentiation conditions. (B) ALP activity was obviously increased in PML-overexpressing hMSCs cultured under osteogenic or non-osteogenic conditions for 7 days. (C) After being cultured under normal conditions for 7 days, ALP mRNA expression was upregulated in PML-overexpressing hMSCs, while ALP mRNA expression was inhibited in PML-RNAi hMSCs. "P<0.05 vs. the two control groups. PML, promyelocytic leukemia; hMSCs, human mesenchymal stem cells; ALP, alkaline phosphatase.

Table I. The most differentially expressed genes between the mock and PML-transfected hMSCs as detected by genome-wide microarray.

\begin{tabular}{|c|c|c|c|c|c|}
\hline \multirow[b]{2}{*}{ Accession no. } & \multirow[b]{2}{*}{ Gene name } & \multirow[b]{2}{*}{ Regulation } & \multicolumn{2}{|c|}{$\begin{array}{l}\text { Normalized intensity } \\
\text { of each group }\end{array}$} & \multirow[b]{2}{*}{ P-value } \\
\hline & & & Mock & PML & \\
\hline NM-022971 & FGFR2 & Downregulation & 280.16 & 97.79 & 0.014 \\
\hline NM-022975 & FGFR2 & Downregulation & 117.37 & 51.71 & 0.017 \\
\hline NM-022976 & FGFR2 & Downregulation & 596.47 & 307.03 & 0.045 \\
\hline NM-000576 & IL-1B & Upregulation & 47.05 & 139.58 & 0.046 \\
\hline NM-000584 & IL-8 & Upregulation & 67.36 & 211.69 & 0.005 \\
\hline J05213 & IBSP & Upregulation & 90.37 & 267.03 & 0.012 \\
\hline
\end{tabular}

PML, promyelocytic leukemia; hMSCs, human mesenchymal stem cells. FGFR2, fibroblast growth factor receptor 2; IBSP, integrin-binding sialoprotein; IL, interleukin.

studies have shown that IBSP is detectable in the early stage of osteogenesis (13). With the increased expression of PML for 3 days, hMSCs cultured in normal conditions (DMEM-LG containing 10\% FBS) exhibited strong upregulation of IBSP. Moreover, IBSP expression was reduced when the PML gene was knocked down for 3 days as compared to the mocktransfected and normal cells ( $\mathrm{P}<0.05$ for all) (Fig. 6).

\section{Discussion}

In the present study, we investigated the effect of PML in hMSCs and found that PML inhibits cell proliferation, yet promotes the osteogenic differentiation of hMSCs.

To date, the biochemical and molecular functions of PML have been studied extensively, and the roles of PML 

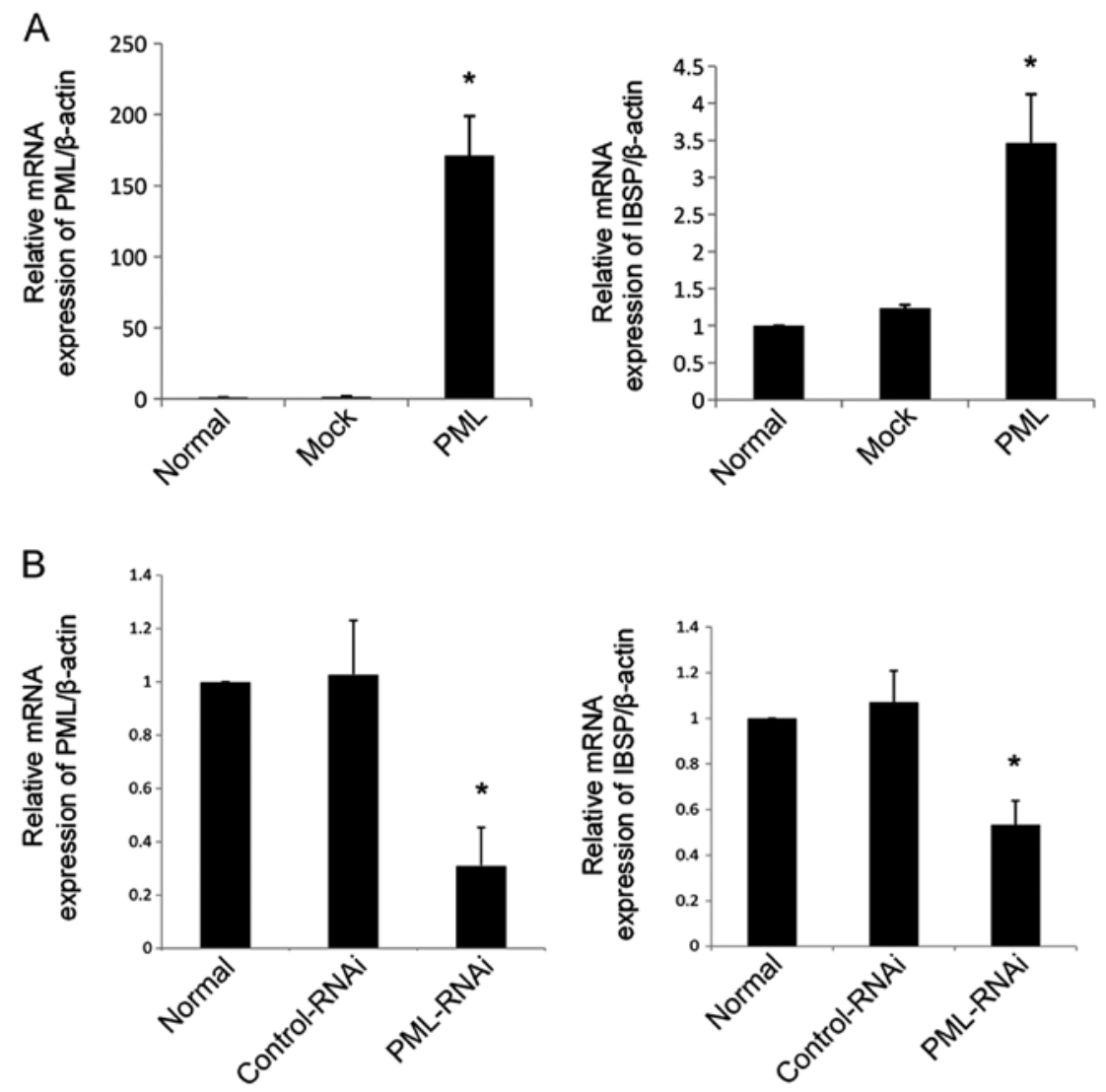

Figure 6. IBSP expression displays the same tendency as PML. (A) Together with the increased expression of PML for 3 days, mRNA of IBSP was upregulated. (B) Following knockdown of the PML gene for 3 days, IBSP expression was reduced. " $\mathrm{P}<0.05$ vs. the two control groups. IBSP, integrin-binding sialoprotein; PML, promyelocytic leukemia.

as a tumor-suppressor in cell proliferation, differentiation, and apoptosis have been shown in many types of tumor cells $(14,15)$. In cancer cells, enhanced PML expression can arrest the cell cycle in the G1 phase (15), and PML overexpression has been shown to induce tumor cell apoptosis via the caspase-dependent pathway (16). Recently, several reports have demonstrated an unexpected and critical role of PML in stem cell and progenitor cell biology. PML is required for HSCs maintenance and NPCs differentiation $(7,9)$. Yet, the role of PML in hMSCs remains unknown.

In the present study, we found that PML was expressed in hMSCs, and the expression was increased time-dependently along with cell osteogenic differentiation. To further investigate the role of PML in hMSCs proliferation and osteogenic differentiation, we established an hMSCs cell line that stably overexpressed PML. PML, as a key tumor-suppressor, can suppress cell growth in many types of cancer cells via a caspase-associated pathway (17). In hMSCs, enhanced PML expression also decreased cell proliferation by inducing caspase-associated apoptosis, which is in accordance with previous studies in tumor cells $(18,19)$. The PML-induced hMSCs apoptosis was verified by the decreased expression of apoptosis-associated proteins, such as pro-caspase- 3 and PARP, and by an increased production of cleaved caspase- 3 as detected by western blot analysis. Cell apoptosis is strongly dependent on cell cycle arrest; however, PML overexpression induced no significant changes to the cell cycle. One possible explanation for this phenomenon is that hMSCs have different cell cycle condition as compared to cancer cells. Cell cycle analysis using hMSCs in normal culture showed that $>90 \%$ of cells were in the G0/G1 phase (20); thus it is difficult to detect cell cycle arrest in hMSCs. Nevertheless, when PML expression was inhibited by RNAi, the proportion of hMSCs in the $\mathrm{S} / \mathrm{G} 2$ phase of the cell cycle had clearly increased. In other words, loss of PML expression was in favor of cell proliferation by promoting cell cycle progression. These results imply that a stable and a certain level of expression of PML plays an important role in maintaining the normal proliferation capacity of hMSCs. When the expression of PML is altered, proliferation of hMSCs is affected.

Calcium deposition is a major marker of osteogenic differentiated hMSCs and is positively correlated with the degree of differentiation. Under osteogenic differentiation conditions, hMSC calcium deposition becomes evident after 7 days (21), as was indicated by von Kossa staining, which is an important tool to examine mineralization in vitro (22). In our study, mineralization and matrix production of hMSCs cultured in osteogenic differentiation medium were increased in comparison to those cultured under non-osteogenic differentiation conditions by day 7. When PML was overexpressed, hMSCs under an osteogenic differentiation condition caused a dramatic increase in calcium deposition when compared to the normal and mock-transfected cells. To determine whether the increased PML level causes osteogenic differentiation, 
hMSCs were cultured under non-osteogenic differentiation condition for 7 days. We also found a significant calcium deposition in the PML-overexpressing cells, when compared with the normal and mock-transfected cells. ALP protein activity is another important marker of early osteogenetic progression (23). We found that ALP protein activity was enhanced by PML overexpression even when the cells were cultured under a non-osteogenic growth condition and ALP expression was coincidental with ALP activity. These results indicated that the osteogenic differentiation potential of hMSCs was enhanced by PML overexpression.

When evaluating the microarray expression data, we found that IBSP was significantly increased in the PML-transfected hMSCs, but no other osteogenic-related gene was noted. IBSP, as an important osteogenic-related gene, is a member of the small integrin-binding ligand N-linked glycoprotein (SIBLING) family and is responsible for mineralization and matrix production $(24,25)$. Upregulation of IBSP gene expression is associated with osteoblast differentiation. cAMP response element (CRE) is an important promoter of the IBSP gene, which is required for gene transcriptional activation (26). The phosphorylation of CRE-binding protein (p-CREB) stimulates IBSP gene expression. However activation of the IBSP gene by $\mathrm{p}$-CREB partly requires co-activation of the CREB-bind protein (CBP) (27). PML, as the major constituent of PML-NBs, has been proven to interact with $\mathrm{CBP}$ in vitro and in vivo. CBP was co-localized with PML in PML-NBs, and upregulation of PML protein has been shown to promote the function of CBP as a potent coactivator (28). When PML was overexpressed in hMSCs, the enhanced activity of CBP stimulated IBSP gene transcription. In the present study, we proved that IBSP expression displayed the same tendency with PML.

In addition, FGFR2 was the most highly downregulated gene in the PML-transfected cells. The fibroblast growth factor (FGF) family comprises 22 proteins and plays a key role in bone formation, homeostasis, and repair through activation of the FGF receptor (FGFR) (29), and the expression of FGFR in hMSCs has been well acknowledged. Thus, in hMSCs, FGFR signaling activation can increase cell proliferation and is essential for cell differentiation $(30,31)$. However, the mechanisms of FGFR2 downregulation caused by PML warrant further investigation.

In conclusion, the present study revealed a previously unknown role of PML in the regulation of cell proliferation and osteogenic differentiation of hMSCs. Briefly, PML regulates hMSCs as an inhibitor of cell proliferation, but promotes osteogenic differentiation, which is associated with the upregulation of IBSP. Thus, our findings offer new insights into the role of PML in hMSC proliferation and differentiation. Future studies will enable us to better discern the functions of hMSCs in order to broaden the spectrum of their applications.

\section{Acknowledgements}

We thank Shanghai KangChen Bio-Tech for their excellent technical assistance with the microarray analysis. This study was supported by the National Natural Science Foundation of China (nos. 81170526, 81100364, 2009C14011), the Major
Science, and the Technology Program of Zhejiang Province (no. Z2100097).

\section{References}

1. Chen BY, Wang X, Chen LW and Luo ZJ: Molecular targeting regulation of proliferation and differentiation of the bone marrow-derived mesenchymal stem cells or mesenchymal stromal cells. Curr Drug Targets 13: 561-571, 2012.

2. Satija NK, Gurudutta GU, Sharma S, et al: Mesenchymal stem cells: molecular targets for tissue engineering. Stem Cells Dev 16: 7-23, 2007.

3. Ling L, Nurcombe V and Cool SM: Wnt signaling controls the fate of mesenchymal stem cells. Gene 433: 1-7, 2009.

4. De Boer J, Wang HJ and Van Blitterswijk C: Effects of Wnt signaling on proliferation and differentiation of human mesenchymal stem cells. Tissue Eng 10: 393-401, 2004.

5. Shtutman M, Zhurinsky J, Oren M, Levina E and Ben-Ze'ev A: $P M L$ is a target gene of $\beta$-catenin and plakoglobin, and coactivates $\beta$-catenin-mediated transcription. Cancer Res 62: 5947-5954, 2002.

6. Scaglioni PP, Yung TM, Cai LF, et al: A CK2-dependent mechanism for degradation of the PML tumor suppressor. Cell 126: 269-283, 2006.

7. Regad T, Bellodi C, Nicotera P and Salomoni P: The tumorsuppressor Pml regulates cell fate in the developing neocortex. Nat Neurosci 12: 132-140, 2009.

8. Salomoni P, Dvorkina M and Michod D: Role of the promyelocytic leukaemia protein in cell death regulation. Cell Death Dis 3: e247, 2012.

9. Ito K, Bernardi R, Morotti A, et al: PML targeting eradicates quiescent leukaemia-initiating cells. Nature 453: 1072-1078, 2008.

10. Meuleman N, Tondreau T, Delforge A, et al: Human marrow mesenchymal stem cell culture: serum-free medium allows better expansion than classical $\alpha$-MEM medium. Eur J Haematol 76: 309-316, 2006.

11. Liu L, Yu Q, Lin J, et al: Hypoxia-inducible factor-1 $\alpha$ is essential for hypoxia-induced mesenchymal stem cell mobilization into the peripheral blood. Stem Cells Dev 20: 1961-1971, 2011.

12. Zhang L, Zheng W, Wang Y and Huang H: Human bone marrow mesenchymal stem cells support the derivation and propagation of human induced pluripotent stem cells in culture. Cell Reprogram 15: 216-223, 2013.

13. Gay IC, Chen S and MacDougall M: Isolation and characterization of multipotent human periodontal ligament stem cells. Orthod Craniofac Res 10: 149-160, 2007.

14. Ferbeyre G, de Stanchina E, Querido E, Baptiste N, Prives C and Lowe SW: PML is induced by oncogenic ras and promotes premature senescence. Genes Dev 14: 2015-2027, 2000.

15. Salomoni P, Ferguson BJ, Wyllie AH and Rich T: New insights into the role of PML in tumour suppression. Cell Res 18: 622-640, 2008.

16. Wang ZG, Ruggero D, Ronchetti S, et al: PML is essential for multiple apoptotic pathways. Nat Genet 20: 266-272, 1998.

17. Li X, Lu Y, Huang W, et al: In vitro effect of adenovirus-mediated human Gamma Interferon gene transfer into human mesenchymal stem cells for chronic myelogenous leukemia. Hematol Oncol 24: 151-158, 2006.

18. Lallemand-Breitenbach V and de Thé H: PML nuclear bodies. Cold Spring Harb Perspect Biol 2: a000661, 2010.

19. Takahashi Y, Lallemand-Breitenbach V, Zhu J and de Thé H: PML nuclear bodies and apoptosis. Oncogene 23: 2819-2824, 2004.

20. Conget PA and Minguell JJ: Phenotypical and functional properties of human bone marrow mesenchymal progenitor cells. J Cell Physiol 181: 67-73, 1999.

21. Pittenger MF, Mackay AM, Beck SC, et al: Multilineage potential of adult human mesenchymal stem cells. Science 284: 143-147, 1999.

22. George J, Kuboki Y and Miyata T: Differentiation of mesenchymal stem cells into osteoblasts on honeycomb collagen scaffolds. Biotechnol Bioeng 95: 404-411, 2006.

23. Ogawa R, Mizuno H, Watanabe A, Migita M, Shimada T and Hyakusoku H: Osteogenic and chondrogenic differentiation by adipose-derived stem cells harvested from GFP transgenic mice. Biochem Biophys Res Commun 313: 871-877, 2004. 
24. Keselowsky BG, Collard DM and García AJ: Integrin binding specificity regulates biomaterial surface chemistry effects on cell differentiation. Proc Natl Acad Sci USA 102: 5953-5957, 2005.

25. Ilmer M, Karow M, Geissler C, Jochum M and Neth P: Human osteoblast-derived factors induce early osteogenic markers in human mesenchymal stem cells. Tissue Eng Part A 15: 2397-2409, 2009.

26. Shimizu E, Matsuda-Honjyo Y, Samoto H, et al: Static magnetic fields-induced bone sialoprotein (BSP) expression is mediated through FGF2 response element and pituitaryspecific transcription factor-1 motif. J Cell Biochem 91: 1183-1196, 2004.

27. Zhang X, Odom DT, Koo SH, et al: Genome-wide analysis of cAMP-response element binding protein occupancy, phosphorylation, and target gene activation in human tissues. Proc Natl Acad Sci USA 102: 4459-4464, 2005.
28. Doucas V: The promyelocytic (PML) nuclear compartment and transcription control. Biochem Pharmacol 60: 1197-1201, 2000.

29. Marie PJ: Fibroblast growth factor signaling controlling bone formation: an update. Gene 498: 1-4, 2012.

30. Ryu E, Hong S, Kang J, et al: Identification of senescence-associated genes in human bone marrow mesenchymal stem cells. Biochem Biophys Res Commun 371: 431-436, 2008.

31. Coutu DL, François M and Galipeau J: Inhibition of cellular senescence by developmentally regulated FGF receptors in mesenchymal stem cells. Blood 117: 6801-6812, 2011. 